\title{
Design and Analysis Sensor Deployment Scheme for Underwater Communication
}

\author{
Shwetha S Y, Mr. Chethan B.R., Dr. Taranath N L, Mr. Darshan L M, \\ $4^{\text {th }}$ Sem M.Tech Student, Dept. of E\&C, \\ PESITM, Shivamogga, \\ Asst. Prof, Dept. of E\& C, \\ PESITM, Shivamogga, \\ Asst. Prof, Dept. of CS \&E, \\ AIT, Chikkamagaluru \\ Asst. Prof, Dept. of CS \&E, \\ AIT, Chikkamagaluru
}

\begin{abstract}
:
At the point while setting up the submerged acoustic sensor system (UASN), hub deployment will be the above all else errand, whereupon numerous basic system administrations, for example, system topology control, directing, \& limit recognition, will be manufactured. Where hub deployment at 2-D physical remote sensor systems had been broadly concentrated, little consideration must be gotten through the 3-D partners.

This paper goes for examining the effects for hub deployment procedures at localization exhibitions at the 3-D environment. All the more particularly, these re-enactments directed at this project uncover which of general tetrahedron deployment plan outflanks these irregular deployment plan \& this 3D shape deployment plan as far as decreasing localization blunder \& expanding localization proportion by keeping up these normal number for neighbouring anchor hubs \& system availability. Given this way which arbitrary deployment will be essential decision for greater part of viable applications for date, the outcomes will be relied upon for reveal some insight onto the outline UASNs sooner rather than later.
\end{abstract}

\section{Introduction}

Underwater sensor systems[1] will be imagined for empowering applications of oceanographic information accumulation, contamination observing, seaward investigation, debacle avoidance, helped route \& strategic observation applications. Numerous unmanned or else selfruling underwater vehicles(UUVs, AUVs), outfitted along underwater sensors, would likewise find application at investigation for common underneath ocean assets \& social affair for experimental information at shared observing missions. Thirds for earth surface will be made out for water. Contrasted \& the person's nature along area, there will be still numerous un-investigated underwater ranges. These need huge examination endeavors.

This examination for Underwater Acoustic Networks[4] (UANs) will be pulling at consideration because for vital applications of underwater such as military \& business purposes. Many research interest \& endeavors will be moving for this zone as of late. These wide utilizations for UANs incorporate still not constrained for: Information trade among hubs which will be inside this scope for system, or else outside the system along assistance for, e.g., an entryway, the switch focus. This essential configuration objective for correspondence systems will be of trading data. At the UAN, trading data amongst hubs will be one for its crucial applications. The case will be which underwater Internet, at which clients could share data without together, will get for reasonable rather than fantasy, if UANs will be conveyed.

Another critical application will be ongoing correspondence along submarines \& self-sufficient underwater vehicles at system arrangements. For case, concise \& helpful versatile inspecting for $3 \mathrm{D}$ waterfront sea environment will be performed through Odyssey-class AUVs[2]. Such sort of exercises could enhance human capacity for watching \& anticipate these attributes for sea/lake/stream environment. This incorporates observation, reconnaissance, and targeting $\&$ interruption discovery. Through utilizing distinctive sorts for sensors, this UAN could accomplish more precise \& arrangement for low mark targets contrasted \& customary observation frameworks.

Contamination at close shore seas will be the critical issue $\&$ needs close watch. UANS could perform various types of contamination observing, e.g., substance, organic, atomic, \& oil spillage contaminations at bayous, lakes, or else waterways. UANs[4] could likewise be utilized for screen sea streams \& temperature change, e.g., the worldwide temperature alteration impact for sea. Underwater investigations will be troublesome of people because for high water weight, erratic underwater exercises \& boundless size of obscure region.

UANs could help us investigate this underwater world which will be not acquainted along. Such sorts of exercises incorporate investigating minerals \& oilfields, deciding schedules of lying under ocean links, \& so forth.

Through sending Acoustic Sensor Networks at remote areas for screening undersea exercises, sea related calamity such as tidal wave \& ocean tremor could caution for waterfront territories continuously while this happens. The UAN could recognize mine effectively through utilizing acoustic sensors \& optical sensors together. An AUV[2] system base will be presented for mine counter measure operations. From the 
correspondence framework angle, underwater environment will be quite different from the ground-based partner.

This project researches effects on various deployment plans for localization exhibitions, for example, localization mistake, localization proportion, normal number of neighboring grapple nodes, \& system availability, at 3-D UASNs. For best of our insight, this will be principal work which looks at these localization exhibitions for irregular deployment, solid shape deployment, \& general tetrahedron deployment plans at 3-D UASNs. These reproductions directed at this project uncover which of standard tetrahedron deployment plan outflanks these irregular deployment plan \& 3D square deployment plan regarding diminishing localization mistake \& expanding localization proportion when keeping up this normal number for neighboring grapple nodes \& system network. This essential decision of vast majority of down for earth applications of the date will be irregular deployment that, at spite for its effortlessness, might bring about an assortment of issues, for example, system parcel, non-uniform system scope, \& so on.

\subsection{Proposed System :}

UASNs will be made out for various types of sensor nodes (i.e., surface sink, underwater sensor nodes, \& so on.) for cooperatively perform observing errands above this threedimensional (3D) space. The three-dimensional UASN engineering will be appeared at Figure 1. UASNs comprise for static sensor nodes that will be conveyed both on water surface \& underwater \& programmed portable sensor nodes for performing community observing errands over the given checked space. Static sensor nodes of most part comprise for detecting gadget, the microcontroller, $\&$ an acoustic handset along the constrained measure for vitality.

Programmed versatile sensor nodes, for example, self-ruling underwater vehicles (AUVs), unmanned underwater vehicles (UUVs), \& low-control lightweight flyers ordinarily have the lot of vitality which could be supplemented while required. As indicated through these application necessities, various types of sensor nodes could be conveyed at UASNs, which will be, surface sinks, underwater nodes, base nodes, \& programmed versatile nodes. Surface sinks will be at charge for information accumulation \& worldwide position framework (GPS) signal securing; surface sinks could be either stationary or versatile.

Underwater nodes will be outfitted along coasting floats that could be expanded through pumps for conforming the profundities for covering this whole observed space. Typically, base nodes will be secured at base for sea to screen these two-dimensional (2D) range or else work together along underwater nodes \& programmed portable nodes for satisfying checking errands at 3D space.

Programmed versatile nodes could get GPS signals when coasting at this sea surface, $\&$ afterward jump for the settled profundity \& move amongst underwater nodes taking following the predefined direction for helping along localization or data social event, et cetera. Occasions will be distinguished through sensor nodes locally \& data will be exchanged for surface sinks through multi-bounces or else programmed portable nodes utilizing acoustic correspondence. At which point, this information could be sent for on shore controlling focuses that have bigger capacity limit of future preparing.

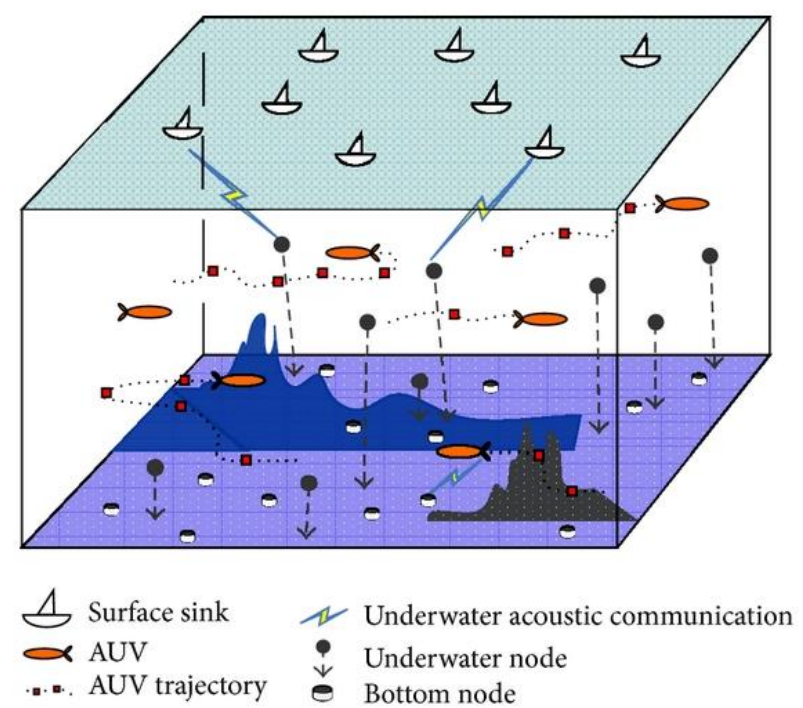

Figure 1: 3D view for Proposed System Architecture. At UASNs, sensor nodes speak along each other through means of acoustic signs, since because one of the kind qualities for underwater acoustic channels (extensive spread deferral, high blunder rate, multi-way impacts, \& so on.), new calculations \& conventions ought for particularly intended for 3D UASNs. At addition, UASNs ordinarily work at dubious \& portable situations wherein freeskimming underwater sensor nodes float gradually along this water momentum. Therefore, this relative movement for transmitter otherwise recipient might make this Doppler impact.

\subsection{Advantages for Proposed System :}

- This could attain minimal delay.

- This could balance consumption of energy throughout simultaneous transmissions.

- This could increase this coverage of network.

- This could attain higher localization accuracy while maintaining better localization ratio, these average number of sensor nodes, \& connectivity of network.

\subsection{Objectives of Paper :}

$>$ To adopt Contingent upon the application necessities, various types of sensor nodes could be conveyed at UASNs,

$>$ To enable Surface sinks implied of information accumulation \& GPS signal obtaining, underwater nodes furnished along gliding floats

$>$ To swelled through pumps for modifying the profundities for guarantying full scope

$>$ Incorporating Programmed versatile nodes, for example, self-governing underwater vehicles, unmanned underwater vehicles, \& low-control lightweight flyers.

\section{Review of Literature}

Manjula.R.B et.al.[2] The seas remain slightest investigated wildernesses of this planet \& numerous maritime \& sea applications appear for generally moderate cutting edge information that correspondence to advancements. This regular \& man-made debacles which has occurred at course for stirred critical enthusiasm for 
checking maritime situations for experimental, natural, business, wellbeing, country security \& military needs.

These underwater environment contrasts as of this physical radio environment both as far as the vitality expenses \& channel proliferation marvels. This underwater channel will be portrayed through long proliferation times \& recurrence subordinate constriction which will be profoundly influenced through separation among nodes \& also through this connection introduction. Some of different issues at that UWSNs vary physical will be restricted data transmission, obliged battery power, more disappointment for sensors on account for fouling \& consumption, \& so on. The paper exhibits the few essential key angles \& models for UWSNs, developing examination issues for underwater sensor systems \& uncovered these scientists onto systems administration for underwater specialized gadgets of energizing sea checking $\&$ investigation applications.

David P. Williams et. al.[4] Another versatile technique of performing information gathering along this sonar-prepared self-governing underwater vehicle (AUV) will be proposed. This methodology will be general as at this will be relevant for an extensive variety for underwater undertakings which depend on resulting preparing for side-looking sonar symbolism. Through keenly apportioning assets \& quickly responding for information gathered at-mission, this proposed approach all the while augments these data content at information \& abatements general study time. The enhancements will be accomplished through adjusting these AUV course for averting parts for mission territory as of being either described through poor picture quality otherwise darkened through shadows brought about through sand swells. This top relationship for continuous sonar returns will be utilized as the measure for picture quality. For recognizing these nearness $\&$ assessment introduction for sand swells, another creative calculation will be produced. These segments for general information driven way arranging calculation will be deliberately developed for allowing quick constant execution along just insignificant AUV on board handling abilities. Test results at light for genuine sonar information gathered adrift will be utilized for exhibiting this guarantee for proposed approach.

Jugen Nie et. $\mathbf{A l}[1]$. The exploration means for creating novel innovations for effectively coordinate remote correspondence systems \& Underwater Acoustic Sensor Networks (UASNs). Surface door deployment will be one for key procedures of interfacing two systems along various channels. At this work, we propose an improvement strategy taking onto account these hereditary calculation of surface portal deployment, outline this novel transmission component-synchronous transmission, \& acknowledge two proficient directing calculations which accomplish negligible postponement \& payload equalization amongst sensor nodes. We promote build up an explanatory model for contemplating these deferral, vitality utilization \& bundle misfortune proportion for system. The reproduction results check this viability for model, $\&$ exhibit the strategy for various door deployments \& this instrument for concurrent transmission could adequately diminish system delay, vitality utilization $\&$ bundle misfortune rate.

\section{System Design}

System design is the design which is plotted for designing the software related items. Here the complete system design is generated and how the modules, sub modules and the flow of the data between them are done is initialized. It explains the architecture which is used for development of the system. It is the base phase for any of the actual implementation process. The errors done here will be reflected in the coming processes.

\subsection{System Architecture:}

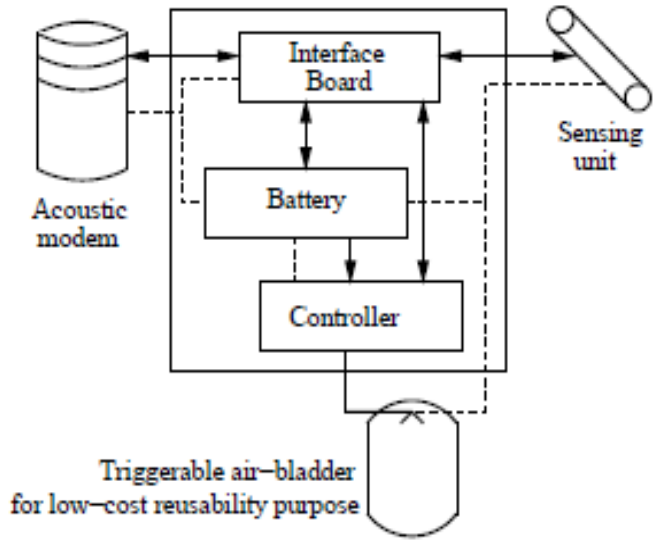

Figure 3.1: THE low-cost underwater sensor node along sensing, acoustic communication \& re-usability capabilities

This huge scale amphibian applications request us for fabricating substantial scale Underwater Ad-hoc Networks (UANET) \& Underwater Sensor Networks (UWSN) for investigating these uninhabited that are present in seas. These distinction amongst UANET \& UWSN will be because of controlled portability \& related execution cost. At UANET, mobile nodes could be actualized via Autonomous Underwater Vehicles (AUV) or else Remotely Operated Vehicles (ROV) that will be high cost robots which could move underneath of water through taking after pre-customized or else self-sufficient movement designs. Be which as this might, these execution expense for such selfmoving nodes will be higher compared to the one for any non-controlled hub. Sooner rather than later, we imagine which of these high-cost unmanned versatile robots would assume vital parts at underwater investigations, for instance at amphibian military crusades.

Then again, UWSN just acquires the small amount for usage expense of UANET in same network scale. All sensor nodes at the UWSN will be of minimal effort (without selfimpelling force). Figure 2 demonstrates this hub design which will be possible nowadays producing capacity. The substantial number for such non-controlled minimal effort sensor nodes could be tossed onto sea for coasting at different profundities of fleeting monitoring, \& could likewise be mounted on seabed or even on anchor for various floats of long haul monitoring.

\subsection{Modules:}

The three deployment schemes performances will be assessed according to the following four criteria:

- Localisation Ratio

- Localisation Error

- Average Number of Neighbouring Anchor Nodes

- Network Connectivity

- Localization ratio will be proportional for quantity of limited conventional nodes for the aggregate 
number of normal nodes. Clearly, these higher localization proportion will be, the more standard nodes could be localized. This localization proportion could be figured as

$$
L_{\text {_ ratio }}=\frac{\mathrm{Nl}}{\mathrm{No}}
$$

Wherein $N l$ will be number of localized nodes that are ordinary, \& No will be total number of ordinary nodes.

- Localization error will be normal separation among these assessed facilitates \& these genuine directions. Obviously, the smaller is the localization error will be, better the localization result will be. The localization mistake could be figured as:

$$
L_{-} \text {error }=\frac{\sum_{i=1}^{N l} \sqrt{\left(u_{i}-x_{i}\right)^{2}+\left(v_{i}-y_{i}\right)^{2}+\left(w_{i}-z_{i}\right)^{2}}}{N_{l}}
$$

Where $(u i, v i, w i)$ will be real coordinates of the ordinary node $i,(x i, y i, z i)$ will be coordinates that are estimated for the ordinary node $i, \& N l$ will be number for localized ordinary nodes.

- Average number of neighbouring anchor nodes will be proportional for quantity of sensor nodes which could speak along stay nodes for aggregate number of sensor nodes. The bigger is the normal number of neighbouring grapple nodes will be, more decisions the standard hub will choose suitable stay nodes for helping along localization. These normal number for neighbouring stay nodes could be processed as takes after:

$$
A_{-} \text {anchor }=\frac{N_{C o m} a}{N}
$$

Wherein Ncom_the will be number of sensor nodes which could communicate along anchor nodes, \& $N$ will be the total number of sensor nodes.

- Network availability will be proportional for quantity of sensor nodes which could speak along other sensor nodes for aggregate number of sensor nodes, which could be registered as takes after:

$$
N_{-} \text {connectivity }=\frac{N c o m}{N}
$$

Where $N$ com will be number of sensor nodes which could communicate along other sensor nodes $\& N$ will be total number of sensor nodes.

\subsection{Specification using Use case diagram}

The graphical depiction of the interactions among the system elements is defined as use case diagram. It is a methodology that is of great significance in system analysis for the identification, clarification and organization of system requirements. The requirements here are mostly the design requirements. Therefore, during system analysis stage, which is done to gather the functionalities the use cases are prepared and the actors are identified. It describes the communication between the actors and the systems during execution of the system.

Fig 5.3 shows the use case diagram for overall system of deployment strategies on localization performance in underwater acoustic sensor networks. Here, in the use case diagram there are four sets of use cases and one actor. Every use case represents the functionality that the system provides. The actor here is involved in the use case by providing the input node as input.

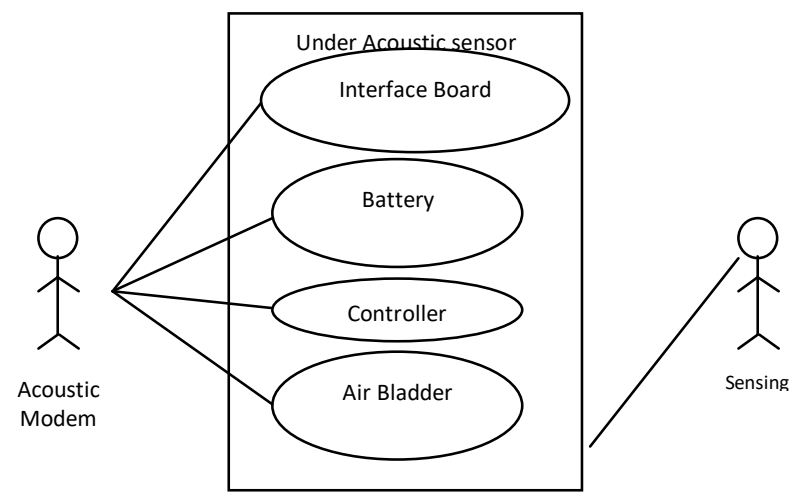

Fig 5.3: Use case diagram for Underwater acoustic networks

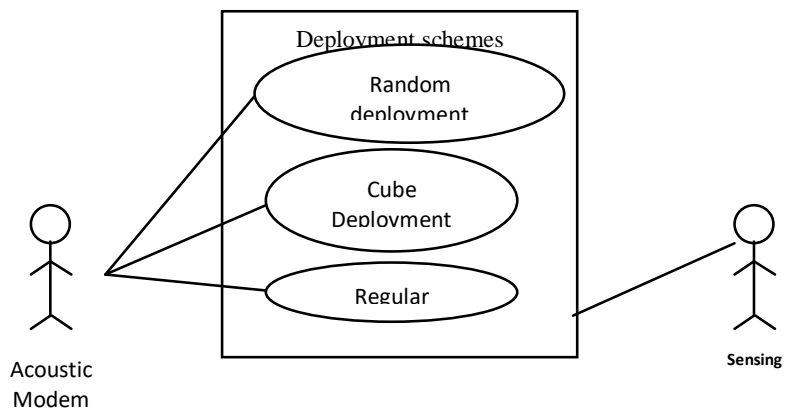

Fig 5.4: Use case diagram for Deployment Schemes In this section, we will illustrate in detail about network model, where the random deployment scheme, the cube deployment scheme, and the regular tetrahedron deployment scheme will be employed. In the random deployment scheme, anchor nodes are deployed randomly in a 3-D monitored region, as shown in Fig 5.4.

As shown in Fig. 5.4 in the cube deployment scheme, anchor nodes are deployed at the vertices of the prepositioned space-filling cubes, whereas in the regular tetrahedron deployment scheme, anchor nodes are deployed at the vertices of some prepositioned regular tetrahedrons. Ordinary nodes in all the three deployment schemes above are deployed randomly in the 3-D monitored space.

\section{Results and Discussions}

4.1 Front-End Design of the Deployment strategies in Underwater Acoustic Sensor Network System :

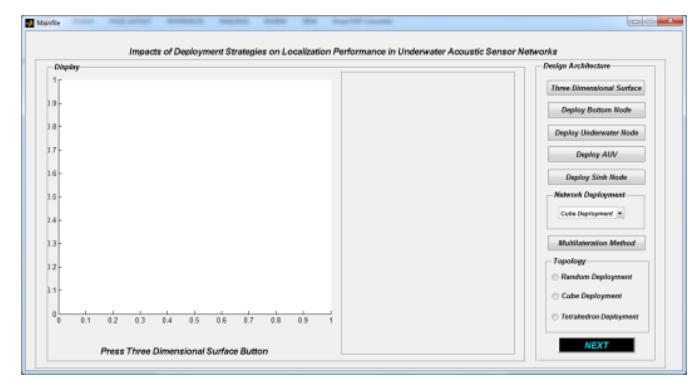

The snapshot 8.1 shows the output page of Underwater Acoustic Sensor Network method. This page consists of few buttons namely, three dimensional surface, deploy bottom node, underwater node, AUV, sink node, Topology and 
Next. Validate button presents the results. It displays localization ratio, network connectivity, average number of nodes, localization error etc., Next button moves the user interface window that is displayed for underwater acoustic sensor networks.

\subsection{Three Dimensional surface in Underwater Acoustic} sensor networks :

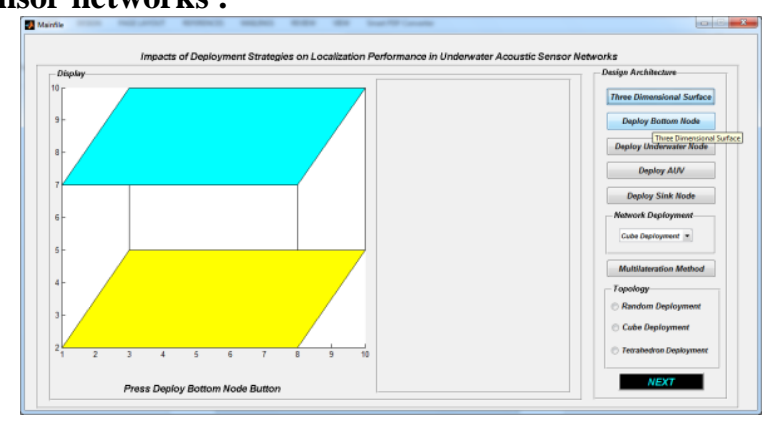

Fig 4.2 shows three dimensional surface in underwater acoustic networks. It will display screen when 3-D surface button is pressed in GUI. It actually displays a cube in which the surface which is not visible to us will be shaded with yellow and green color. This will give an indication to the user that we are deploying a 3-D strategy on Localization Performance in Underwater Acoustic Sensor Networks.

4.3 Creation of bottom nodes for Three Dimensional surface in Underwater Acoustic sensor networks :

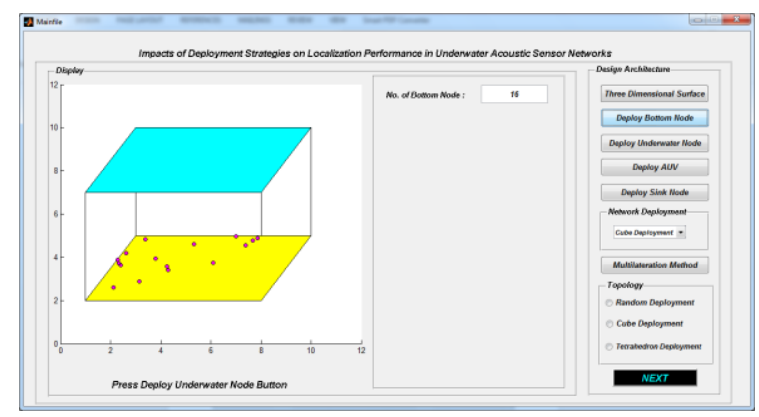

Fig 4.3 shows creation of bottom nodes with 3-D dimensional surface in underwater acoustic networks. It will display number of bottom nodes which actually involved in transmission. The different nodes are indicted by pink color within the network. This will give an indication to the user that about number of bottom nodes which involved in the transmission.

4.4 Selection of different network deployment in Underwater Acoustic sensor networks :

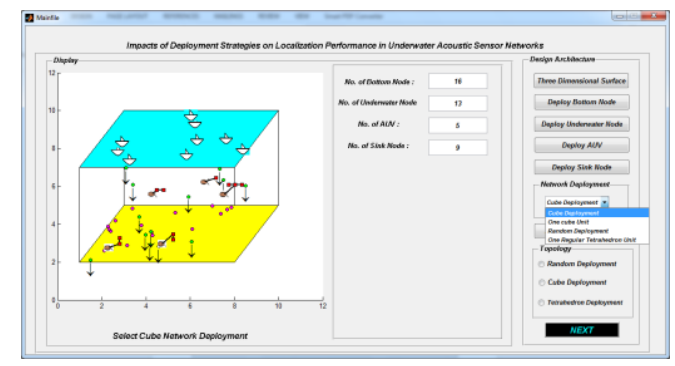

Fig 4.4 shows number of number of sink nodes which involved in 3 Dimensional surface in underwater acoustic networks. It will display the different strategies used to deploy sink node. This Fig illustrates cube deployment strategy used as a network deployment activity.

4.5 Selection of Cube deployment Scheme in Underwater Acoustic sensor networks :

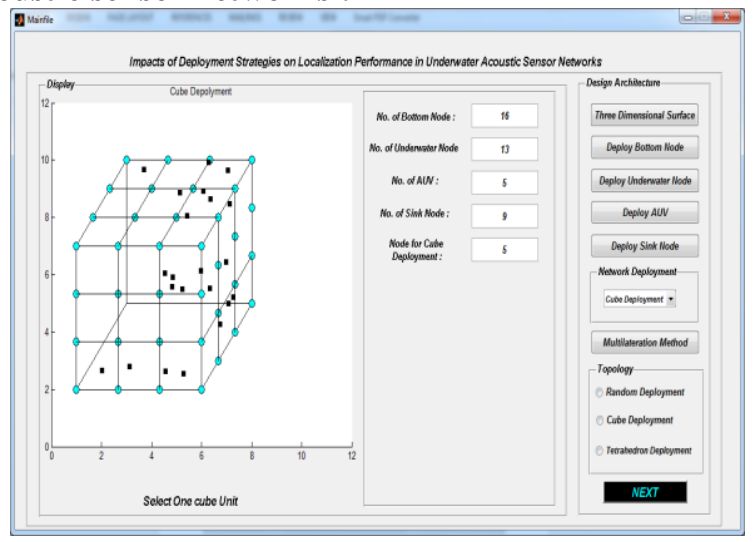

Fig 4.5 shows number of number of sink nodes which involved in 3 Dimensional surface in underwater acoustic networks. It will display the different strategies used to deploy sink node. This Fig illustrates 5 cube deployment nodes are considered for the construction of network.

\subsection{Performance Evaluation in Underwater Acoustic} sensor networks :

Fig 4.6 shows perform evaluation of 3 Dimensional surfaces in underwater acoustic networks. It will display the different metrics used for deployment in underwater acoustic networks. This Fig illustrates anchor node percentage may be set to $6.75 \%, 16 \%$ and $20 \%$ respectively for finding the performance of the network. Once user select anchor node percentage, system shows a dialog box for the indication of processing.

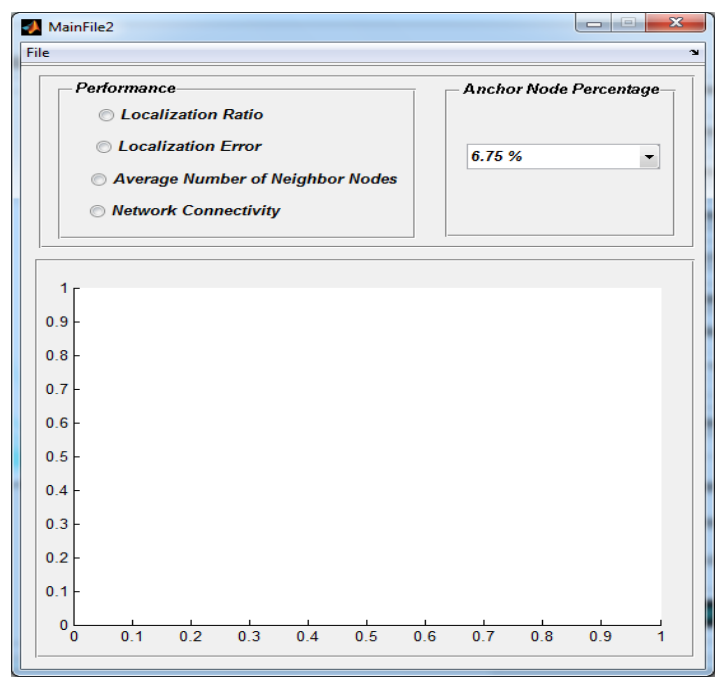

4.7 Performance Evaluation with Localization Ratio in Underwater Acoustic sensor networks : 


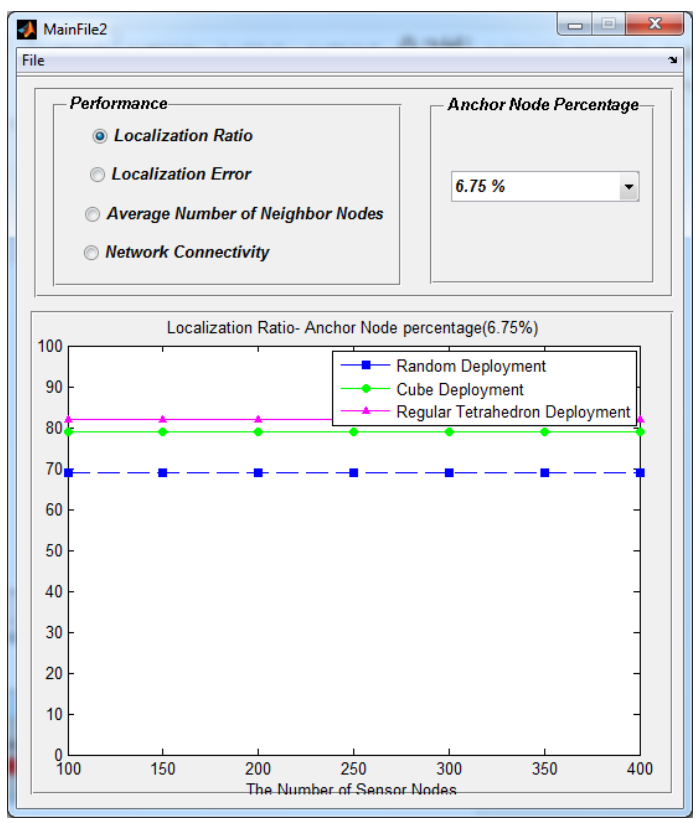

Fig.4.7 depicts the relationship between the localization ratio and the number of sensor1nodes. The regular tetrahedron deployment scheme outperforms both the random deployment scheme and the cube deployment scheme in terms of the localization ratio. When the anchor node percentage is relatively small $(6.75 \%)$, the localization ratio of the random deployment scheme fluctuates more than that of the cube deployment scheme and the regular tetrahedron deploy-ment scheme, as shown in Fig. This is due to the fact that, in the random deployment scheme, some of the edge nodes and isolated nodes will only receive a limited number of coor-dinate messages from anchor nodes to help with localization. On the other hand, as the anchor node percentage increases, the fluctuation of the random deployment scheme decreases rapidly, and the localization ratios of 1the cube deployment scheme and the regular tetrahedron deployment1scheme are nearly the same.

\subsection{Performance Evaluation with Localization Ratio in Underwater Acoustic sensor networks :}

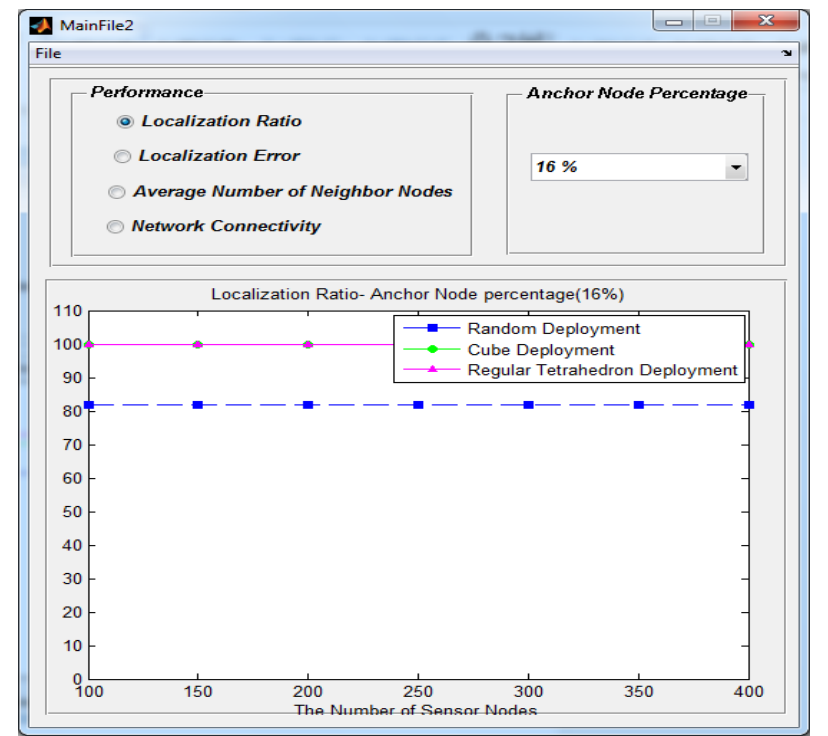

Fig 4.8 shows, when the anchor node percentage is $16 \%$, and the number of sensor nodes is 350, the localization ratio of the cube deployment scheme is $98.75 \%$, and the local ratio of the regular tetrahedron deployment scheme is $100 \%$, as depicted in Fig., because most of the ordinary nodes can be localized when there are enough anchor nodes in the network.

\subsection{Performance Evaluation with Localization Ratio in Underwater Acoustic sensor networks :}

By comparing Fig. 4.9, we conclude that the more anchor nodes there are, the higher the localization ratio will be. For example, if the anchor node percentage is $6.75 \%$, the localization ratio of the regular tetrahedron deployment is $86.06 \%$ when the number of sensor nodes is 150; however, if the anchor node percentage is $16 \%$, the localization ratio of the regular tetrahedron deployment can reach $100 \%$ with the same number of sensor nodes.

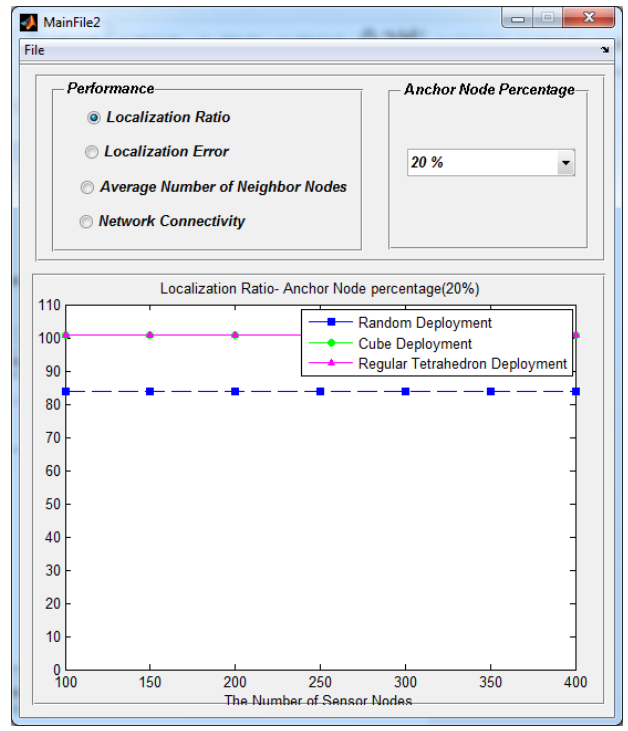

However, when the anchor1node percentage is large enough, the localization ratio reaches a relatively high value and will not change much with the increase in the anchor node percentage. This suggests that, in sparse UASNs, we can increase the number of anchor nodes to achieve higher localization ratio.

\section{Conclusion and Future Enhancement 5.1 Conclusion}

At this framework, we explored a basic issue for hub deployment at UASNs. We proposed three deployment plans at 3-D UASNs (these irregular deployment, 3D square deployment, \& normal tetrahedron deployment plans) \& look at the exhibitions at point of interest regarding localization proportion, localization blunder, normal number of neighbouring stay nodes, \& network availability. Affirming which normal conviction will be suitably picked hub deployment methodology will be for focal significance of exact localizations at UASNs, these reproductions led at this project uncovered which these customary tetrahedron deployment plan beats other two plans as far as lessening localization blunder \& expanding localization proportion, by keeping up these normal number of neighbouring stay nodes $\&$ the sensible network availability.

\subsection{Future Scope}

- $\quad$ The normal subsequent heading will be the top to bottom quantitative treatment for three 
proposed deployment plans following this action would offer many bits of knowledge along respect for this configuration for 3-D UASNs.

- The reasonable model ought to consider this portability for sensor nodes underneath genuine water conditions.

- Most of the current existing hub deployment calculations expect the protected \& dependable environment, wherein, at numerous genuine applications, will be scarcely these cases.

- $\quad$ As sensor nodes underwater will be costly $\&$ these expenses rapidly ascend the profound water, deployment calculations of versatile stay nodes will be relied upon for assume this urgent part at decreasing expenses at profound water situations.

\section{REFERENCES}

[1] "Impacts of Deployment Strategies onLocalization Performance in UnderwaterAcoustic Sensor Networks" by Guangjie Han, Member, IEEE, Chenyu Zhang, Lei Shu, Member, IEEE andJoel J. P. C. Rodrigues, Senior Member, IEEE in IEEE TRANSACTIONS ON INDUSTRIAL ELECTRONICS, VOL. 62, NO. 3, MARCH 2015.

[2] H. Tan, R. Diamant, W. K. G. Seah, \& M. Waldmeyer, "The survey of techniques \& challenges at underwater localization," Ocean Eng., vol. 38, no. 14/15, pp. 1663-1676, Oct. 2011.

[3] R. B. Manjula \& S. S. Manvi, "Issues at underwater acoustic sensor networks," Int. J. Comput. Electr. Eng., vol. 3, no. 1, pp. 1793-8163, Feb. 2011.

[4] G. Han, C. Zhang, L. Shu, N. Sun, \& Q. Li, "The survey on deployment algorithms at underwater acoustic sensor networks, international journal of distributed sensor networks," Int. J. Distrib. Sens. Netw., vol. 11, p. 2013, 2013, Art. ID 314049.

[5] D. P. Williams, "AUV-enabled adaptive underwater surveying for optimal data collection," Intell. Serv. Robit., vol. 5, no. 1, pp. 33-55, Jan. 2012.

[6] N. S. N. Ismail, L. E. Hussein, \& S. H. S. Ariffin, "Analyzing the performance of acoustic channel at underwater wireless sensor network (UWSN)," at Proc. Int. Conf. AMS, Might 2010, pp. 550-555.

[7] R. C. Luo \& O. Chen, "Mobile sensor node deployment \& asynchronous power management for wireless sensor networks," IEEE Trans. Ind. Electron., vol. 59, no. 5, pp. 2377-2385, Might 2012.

[8] B. Huang, C. Yu, \& B. D. O. Anderson, "Understanding error propagation at multihop sensor network localization," IEEE Trans. Ind. Electron., vol. 60, no. 12, pp. 5811-5819, Dec. 2013. 